\title{
Spring coil retraction in coil occlusion of persistent ductus arteriosus
}

\author{
T Ino, K Nishimoto, M Okubo, K Akimoto, K Yabuta, S Kawasaki, Y Hosoda, M Iwahara
}

\begin{abstract}
Aims-To present the short and intermediate term results of coil occlusion of persistent ductus arteriosus and the results of radiographic measurements of spring coils implanted to treat patent ducts.

Patients-22 children underwent coil occlusion. Their ages ranged from 2 years 9 months to 12 years 10 months (mean (SD) age, 6.5 (3.6) years). The duct diameter ranged from 1.0 to $3.5 \mathrm{~mm}$ at the narrowest point (mean $2.6(0.7) \mathrm{mm}$ ). In 11 of the children regular coils were implanted using the non-attached system, while in the other 11 the detachable coil embolisation system was used.
\end{abstract}

Results-12 children (55\%) had no significant residual leaks immediately after procedures involving a single coil delivery. The remaining $10(45 \%)$ had residual leaks immediately after the procedure, although no patient with a large duct showed residual leakage 18 months after the procedure. Radiographic measurement of the coils showed that all implanted coils retracted to $65-85 \%$ of their original size immediately after occlusion. This retraction was more evident in patients showing spontaneous closure of the residual shunt or having a coil $8 \mathrm{~mm}$ in diameter.

Conclusions-Coil embolisation is an acceptable method for occluding persistent ductus arteriosus. Retraction of implanted coils is common in the follow up period. Such retraction may be related to spontaneous closure of residual shunt after embolisation.

(Heart 1998;80:327-329)

Paediatrics, Juntendo

University School of

Medicine, 2-1-1

Hongo, Bunkyo-ku,

Tokyo 113, Japan

$\mathrm{T}$ Ino

K Nishimoto

M Okubo

K Akimoto

K Yabuta

Department of

Thoracic Surgery,

Juntendo University

School of Medicine

S Kawasaki

Y Hosoda

M Iwahara

Correspondence to: Dr T Ino, Department of

Paediatrics, Kameda Medical Centre, 929 Higashi-cho, Kamogawa, Chiba 296-8602, Japan.

Accepted for publication 5 June 1998 occlusions performed in our institute and the results of radiographic measurements of spring coils implanted to treat persistent ductus arteriosus.
Methods

PATIENTS

Between October 1994 and January 1996, 30

consecutive patients with persistent ductus arteriosus were enrolled into the protocol. Of these, 22 have complete follow up data during 18 months after the embolisation procedure. The patients ranged in age from 2 years 9 months to 12 years 10 months (mean (SD) age, 6.5 (3.6) years). Their weights ranged from 10.4 to $40.2 \mathrm{~kg}$ (mean 22 (11) kg). There were 10 boys and 12 girls. Twenty one patients had an isolated persistent ductus arteriosus and the remaining patient had coarctation of the aorta and a ventricular septal defect. In this patient, balloon coarctation angioplasty had been performed at the age of 1 year.

MODIFIED OCCLUDING COIL

Between October 1994 and June 1995, we used a non-attached system with Dacron stranded, stainless steel coils (Cook Inc, Bloomington, Indiana, USA). We increased the length of the coil with a helical diameter of $5 \mathrm{~mm}$ (MWCE-38-8-5) from $5 \mathrm{~cm}$ to $8 \mathrm{~cm}$ and the $8 \mathrm{~mm}$ diameter coil (MWCE-38-12-8 or MWCE-38-15-8) from $8 \mathrm{~cm}$ to 12 or 15 $\mathrm{cm} .{ }^{9}$ These long coils made more than five helical loops when fully released. In July 1995, the Jackson detachable coil embolisation system (Cook Inc) became available in our institution. $^{9}$

PROTOCOL OF THE OCCLUSION PROCEDURE The procedure of the coil implantation was performed as described previously. ${ }^{5-9}$ In the Jackson detachable system, the delivery wire and coil were firmly connected by using the screw shaped system. This system allows the coil to be detached by turning the delivery wire after confirming that it has been fixed in the duct. Ten minutes after releasing the coil, aortography was repeated. After this procedure, the patients were allowed to recover in the paediatric ward. Colour flow imaging studies were performed to look for residual shunts the morning after the procedure. At this time, chest $x$ rays and ECG were repeated. Patients were discharged on the following day.

FOLLOW UP EVALUATION BY COLOUR FLOW IMAGING

An outpatient follow up examination was performed one week, and one, three, six, 12, and 18 months after the procedure. On each visit, a cardiovascular examination was performed, which included chest $x$ rays, an ECG, and a colour flow imaging study. Each patient was subject to a complete echocardiographic study, including colour flow imaging, 
A

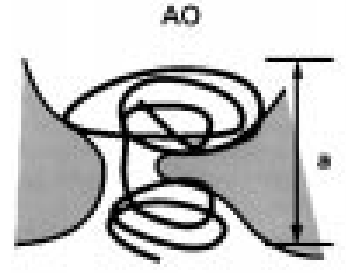

PA
日

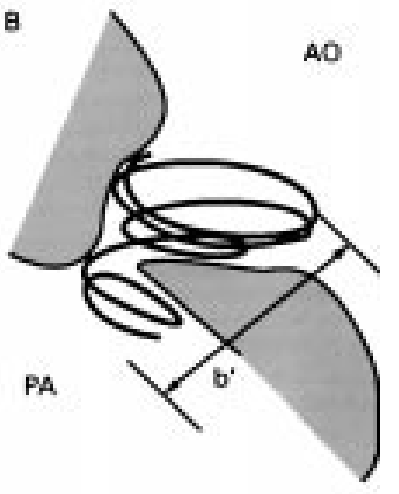

Anteroposterior view
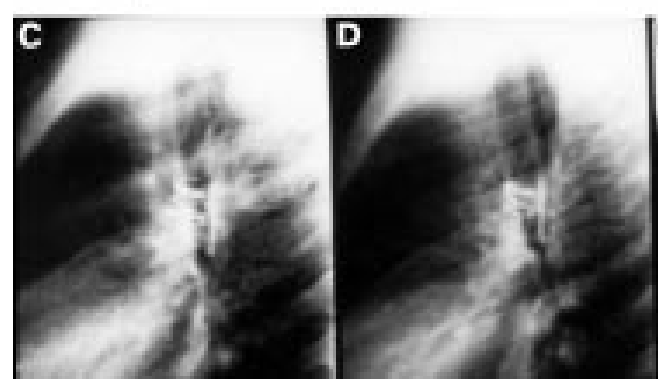

Lateral view

Figure 1 Scheme of quantitative measurement of implanted coil. On the anteroposterior view $(A)$, the distance between the upper and lower margins of the coils was measured (a). On lateral view (B), the longest distance was measured (b). The mean value $[(a+b) / 2]$ was calculated. AO, aorta; $P A$, pulmonary artery. Chest radiographs show an implanted coil of $5 \mathrm{~mm}$ in diameter immediately after occlusion (C), at six months (D), and at 12 months (E). Coil retraction is evident.

to assess residual ductal flow in the main pulmonary trunk, left pulmonary arterial stenosis, and aortic stenosis, using previously reported techniques. ${ }^{10} 11$

QUANTITATIVE MEASUREMENT OF THE SPRING COIL WITH CHEST RADIOGRAPHY

To assess the relation between the spontaneous closure of residual shunts and the coil configuration following occlusion, quantitative measurement of coil implanted was performed in anteroposterior and lateral views on chest $x$ rays. The distance between one edge of the coil loop and another was measured, as shown in fig 1. On anteroposterior projection the distance between upper and lower margins of the coils (a) was measured, and on lateral projection the greatest distance between the coil loops in the aortic and pulmonary ends (b) was also measured. The mean of (a) and (b) was calculated, and this value on follow up $x$ rays was expressed as a percentage of each value immediately after occlusion ( $\%$ retraction value). Serial measurements were performed on follow up chest $x$ rays at each visit. If two coils were delivered, the longest distance between one edge and another edge was also measured. To obtain an accurate measurement of anteroposterior and lateral chest $x$ rays, the angle produced by the centre lines of trachea and ribs overlapping the coil was measured as a reference. If this angle was beyond $\pm 5^{\circ}$, a further chest $x$ ray was obtained or the result was excluded from the quantitative measurement.

STATISTICAL ANALYSIS

The age, body weight, and minimum diameter of the patent duct were expressed as mean (SD). The prevalence of residual shunts and the \% retraction values were evaluated by actuarial analysis. A Wilcoxon test was used to compare the \% retraction value and coil diameters of patients with and without residual shunts or spontaneous closure. A probability (p) value of $<0.05$ was considered significant.

\section{Results}

ACUTE RESULTS OF COIL OCCLUSION

The smallest internal duct diameters were 1.0 to $3.5 \mathrm{~mm}$ (average $2.6(0.7) \mathrm{mm}$ ). Angiographic classification ${ }^{12}$ revealed a core shaped aortic ampulla (type A) in 14 of the 22 patients, a tubular duct (type C) in three, and a long duct with remote narrowest area (type E) in five. Twelve patients had complete closure with size MWCE-38-8-5, and three patients each with MWCE-38-15-8 and MWCE-38-10-8. Five patients required two coils simultaneously. Complete occlusion was confirmed in 12 of the 22 patients $(55 \%)$ by aortography 10 to 15 minutes after the procedure and by colour flow imaging. The remaining 10 patients (45\%) had persistent residual leakage. Postimplantation aortograms revealed small residual leaks in only five of these 10 patients, and colour flow imaging performed on the same day revealed trivial or trace leakage in the other five patients. The only complication encountered was a coil migrating into the distal left pulmonary artery in one patient, in whom the migrated coil was retrieved successfully and another coil implanted.

FOLLOW UP RESULTS OF RESIDUAL LEAKAGE The follow up period ranged from 18 to 32 months (mean (SD), 23 (8) months). For all 10 patients with immediate residual leakage, no shunt flow was detected by colour flow imaging during the 3-18 month follow up period. Spontaneous closure of residual shunts was confirmed in these patients. One patient had a recanalisation six months to one year after embolisation but had complete occlusion due to spontaneous closure 18 months after the procedure. Actuarial analysis therefore showed a \% residual shunt (number of residual shunts/ total number of patients) of $0 \%$ after 18 months.

\section{RESULTS OF COIL MEASUREMENTS ON CHEST} $x$ RAYS

The angle between the centre line of trachea and rib ranged from $67^{\circ}$ to $89^{\circ}$ (median $83^{\circ}$ ). In all 22 patients, this angle was within $\pm 5^{\circ}$ throughout the follow up period and therefore all chest $x$ rays were retrospectively available for measurement. In all patients with single or double coils, the \% retraction value gradually decreased during the follow up period. This value (mean (SD)) was 95(2)\% after one week, $93(3) \%$ after one month, $88(7) \%$ after three months, $79(7) \%$ after six months, and $72(13) \%$ after 12 months. There was a significant positive correlation between the \% retraction value after six months and the initial coil 


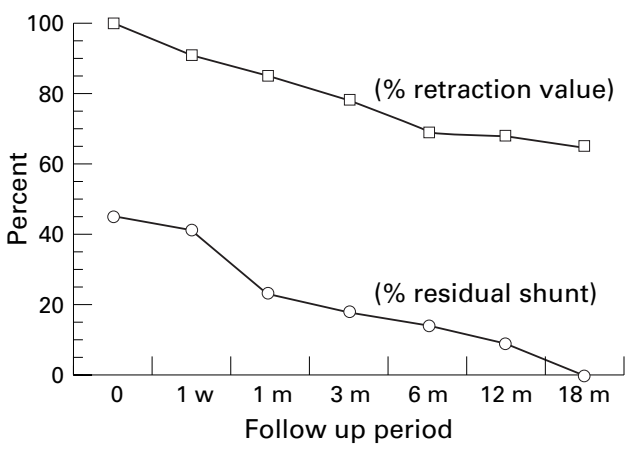

Figure 2 The prevalence of \% residual shunt and the \% retraction value in the follow up period, assessed by actuarial analysis.

size $(r=0.76, \mathrm{p}<0.005)$. In four patients with a single coil of $8 \mathrm{~mm}$ in diameter, the \% retraction value after one month and six months was more evident than in those with a single coil of $5 \mathrm{~mm}$ in diameter $(88(5) \%$ v $95(4) \%$ after one month, $\mathrm{p}<0.05 ; 76(12) \%$ v 82(9)\% after six months, $\mathrm{p}<0.01)$. In addition, the $\%$ retraction values of a single coil from one month to 12 months of follow up were significantly greater in patients with residual shunts than in patients with complete occlusion immediately after the procedure (fig 2).

\section{Discussion}

Since the first reports by Cambier et al in 1992 describing percutaneous closure of persistent ductus arteriosus with spring coils, ${ }^{6}$ the technique has been used by several other workers. ${ }^{5-9} 1314$ The technique is considered to be safe and effective, particularly in the case of small ducts measuring less than $2.5-3.0 \mathrm{~mm}$ in diameter, while a recent investigation has shown that the multiple coil technique could be used to completely occlude a large duct with a diameter of more than $3.0 \mathrm{~mm} \cdot{ }^{7-9}$ To our knowledge, however, there has been no report on coil retraction following coil embolisation for closure of persistent ductus arteriosus. Our results indicate that all coils implanted in the patent duct retract to approximately $70 \%$ of their original size during the 12 months after coil occlusion. This retraction may be related to the interaction between velocity of endothelialisation (fibrous tissue retraction) and thrombus formation, and a retracting force parallel to the long axis of the coil. The greater the initial size of the implanted coil immediately after the procedure, the greater will be the retraction. This indicates that coils with more stretch may subsequently have greater retraction. However, the actuarial analysis curves of the $\%$ residual shunt and the $\%$ retraction value were not correlated. Despite these negative results, our study suggests that coil retraction may be related to the histological mechanism of vascular response following coil occlusion. Acute incomplete thrombus formation in the coil loop first occurs immediately after embolisation, resulting in residual leakage. However, retraction and changes in the position of the coil may lead to new thrombus formation and complete occlusion. In addition, coil retraction in the left pulmonary artery is potentially advantageous in that it may help to avoid left pulmonary arterial stenosis in the long term, even if a larger coil of $8 \mathrm{~mm}$ diameter is implanted. However, this study only followed the natural history of the spring coil in the short term. A long term follow up study is necessary to clarify whether such retraction will ultimately prove advantageous.

Recent investigations have shown that recanalisation rarely occurs after coil embolisation in cases of the aortopulmonary collateral artery and persistent ductus arteriosus. ${ }^{915}$ In our study series, however, only one patient experienced recanalisation. The mechanism of recanalisation remains unclear, although minor changes in coil position and thrombolysis or clot retraction have been suspected by Shim et $a .^{8}$ The results of our study support their hypothesis and suggest that recanalisation may occur owing to the delayed dynamic phase of thrombus maturation, with coil retraction until the coil loop is completely covered by a neoendothelial layer.

In conclusion, both non-attached and detachable systems may be an effective device to ensure complete occlusion in cases of persistent ductus arteriosus. Additionally, coils implanted in patent ducts retract to $65-85 \%$ of their original size immediately after occlusion. This may affect the endothelialisation process involved in the spontaneous closure of the residual shunt after embolisation.

This study was supported in part by grant No C-07670896 from Japan's Ministry of Health and Welfare.

1 Porstmann W, Wierny L, Warnke $\mathrm{H}$, et al. Catheter closure of patent ductus arteriosus: long-term results of 208 cases treated without thoracotomy. Radiol Clin North Am 1971;9:203-18.

2 Rashkind WJ, Cuaso CC. Transcatheter closure of patent ductus arteriosus; successful use in a 3.5 kilogram infant. Pediatr Cardiol 1979;1:3-7.

3 Khan MA, Yousef SA, Mullins CE, et al. Experience with 205 procedures of transcatheter closure of ductus arteriosus in 182 patients, with special reference to residual shunts and long-term follow-up. If Thorac Cardiovasc Surg 1992;104:1721-7.

4 Benson LN, Freedom RM. Balloon dilatation of the very small patent ductus arteriosus in preparation for transcatheter occlusion. Cathet Cardiovasc Diagn 1989;18:48-9.

5 Moore JW, George L, Kirkpatrik SE, et al. Percutaneous closure of the small patent ductus arteriosus using occluding spring coils. F Am Coll Cardiol 1994;23:759-65.

6 Cambier PA, Kirby WC, Worthham DC, et al. Percutaneous closure of the small $(<2.5 \mathrm{~mm})$ patent ductus arteriosus using coil embolization. Am 7 Cardiol 1992;69:815-16.

7 Hijazi ZM, Geggel RL. Results of anterograde transcatheter closure of patent ductus arteriosus using single or multiple
clion closure of patent ductus arteriosus using single
Gianturco coils. Am 7 Cardiol 1994;74:925-9.

8 Shim D, Fedderly RT, Beekman RH, et al. Follow-up of coil Shim D, Fedderly RT, Beekman RH, et al. Follow-up of coil
occlusion of patent ductus arteriosus. $\mathcal{F}$ Am Coll Cardiol 1996;28:207-11

9 Ino T, Yabuta K. Is multiple coil embolization feasible? [letter] Am f Cardiol 1995;76:637.

0 Sreeram M, Miller P, Walsh KP. Lung perfusion studies following detachable coil occlusion of the arterial duct [abstract]. Circulation 1996;94:I-482.

11 Smallhorn JF, Huhta JC, Anderson RH, et al. Suprasternal cross-sectional echocardiography in assessment of patent ductus arteriosus. Br Heart f 1982;48:321-30.

12 Krichenko A, Benson LN, Burrows P, et al. Angiographic classification of the isolated, persistently patent ductus arteriosus and implications for percutaneous catheter occlusion. Am f Cardiol 1989;63:877-80.

13 Sommer RJ, Gutierrez A, Lai WW, et al. Use of preformed nitinol snare to improve transcatheter coil delivery in occlusion of patent ductus arteriosus. $\mathrm{Am} \mathcal{f}$ Cardiol occlusion of pate

14 Lloyd TR, Fedderly R, Mendelsohn AM, et al. Transcatheter occlusion of patient ductus arteriosus with Gianturco coils. Circulation 1993;88:1412-20.

15 Perry SB, Radtke W, Fellows KE, et al. Coil embolization to occlude aortopulmonary collateral vessels and shunts in patients with congenital heart disease. $\mathcal{F} \mathrm{Am}$ Coll Cardiol 1989;13:100-8. 